\title{
Percepções sociais de pacientes sobre profissionais de saúde e outros estressores no ambiente de unidade de terapia intensiva
}

\author{
Patients' social perceptions about health professionals \\ and other stress factors in an intensive care unit environment
}

Leonardo Mello de SOUSA

Edson Alves de SOUZA FILHO

\section{Resumo}

\begin{abstract}
Observaram-se as percepções sociais de pacientes a respeito de profissionais como minimizadores ou maximizadores de stress experimentado em Unidade de Terapia Intensiva. A situação em Unidade de Terapia Intensiva facilita o aparecimento de fenômenos que alteram as percepções subjetivas no modo de lidar com os assuntos de saúde/doença. Aplicou-se um inventário de avaliação de estressores em Unidade de Terapia Intensiva que continha perguntas sobre quem seria o responsável pelo stress experimentado. Inferiram-se relações entre profissionais da equipe de saúde e dimensões da experiência: individual (paciente), interindividual (paciente e outras pessoas) e intergrupal (paciente e profissionais). Foram 85 participantes; utilizaram-se correlações de Pearson e qui-quadrado. Os aspectos mais estressantes foram os ligados à dimensão individual, enquanto os menos estressantes estiveram relacionados à estrutura física e relações intergrupais. A figura considerada mais estressante foi a "equipe", indicando certa diluição de responsabilidade. Houve mais ênfase nos aspectos físicos do que psicossociais na Unidade de Terapia Intensiva.
\end{abstract}

Unitermos: Estressores. Percepção social. Profissionais. Unidade de terapia intensiva.

\begin{abstract}
We observed patients'social perceptions about professionals as minimizers/maximizers of stress experienced in an Intensive Care Unit. Our assumption was that the situation of an Intensive Care Unit contributes to the appearance of phenomena that change subjective perceptions in order to deal with health/disease matters. The method consisted of applying a questionnaire to evaluate stressful factors in an Intensive Care Unit, and it included certain questions about who would be responsible for provoking the specific stresses experienced. We analyzed relationships between these professionals and some of the dimensions of that experience: individual (patient), inter-individual (patient and other people) and intergroup (patient and professionals). There were 85 participants and we employed the Pearson's correlation and chi-square tests. The most stressful aspects were those related to the individual dimension, while the least stressful were related to physical structure and intergroup relations. We observed that the team was the figure most associated with stress, which points to a certain dilution of responsibility. There was more emphasis on the physical than the psychosocial aspects in an Intensive Care Unit.
\end{abstract}

Uniterms: Stress agents. Social perception. Stressors. Professional identity. Intensive Care Units.

1 Universidade Federal do Rio de Janeiro, Instituto de Psicologia, Programa de Pós-Graduação em Psicologia. Av. Pasteur, 250, fundos, $2^{\circ}$ andar, Praia Vermelha, 22290-902, Rio de Janeiro, RJ, Brasil. E-mail:<leopsi@ibest.com.br>. 
As Unidades de Terapia Intensiva (UTI) têm sofrido mudanças tecnológicas, profissionais e estruturais nesses últimos tempos. Essas unidades especiais começaram a ser criadas em 1952, na Dinamarca, e 1955, nos EUA, que enfrentavam, na época, estados críticos de epidemias. No Brasil, não se sabe ao certo quando foram implantadas as primeiras UTI.

As UTI são usualmente vistas como lugares sombrios, nos quais o fim geralmente é a morte. Seu estereótipo é ratificado por toda uma série de conhecimentos sociais e do senso comum, que são disseminados socialmente. Assim, a internação em UTI, invariavelmente, implica em uma situação de grande risco. Sentimentos como medo, ansiedade, agitações psicomotoras, stress, depressão, abandono, desamparo, dependência, culpa, morte, entre outros, são comumente presentes (Torres, 1998). Nesse sentido, existem trabalhos (Aronson, Akert \& Wilson, 2002; Biaggi, 2002) que sustentam o argumento de que diferenças individuais podem alterar esse quadro a partir de estratégias de enfrentamento a serem adotadas pelos pacientes. Contudo, outros conteúdos de ordem psicossocial poderiam ser considerados, para fins de análise. Para tanto, um trabalho de pesquisa básico é necessário, a fim de conhecer certos aspectos epidemiológicos, psicológicos e psicossociais que circundam esses fenômenos, para a elaboração de políticas preventivas a serem estabelecidas em ambientes hospitalares e similares.

Alguns autores têm chamado a atenção para as percepções sociais da experiência de saúde/doença (Flick, 1993; Herzlich \& Pierret, 1988; Parson, 1951). Tratase de levar em conta os conteúdos mentais e comportamentais (re)construídos por indivíduos, internamente, e em práticas cotidianas no ambiente hospitalar, para lidar com os processos de saúde e doença vividos (Flick, 1993; Herzlich, 1973). Especificamente, diante de situações que ocorrem em UTI, um trabalho perceptivo individual e social deve ocorrer entre os pacientes para restaurar a estabilidade psicológica e psicossocial comprometida (Wagner, Duveen, Themel \&Verma, 2000). Parte da estabilização buscada decorre da atribuição de responsabilidade a diferentes personagens da equipe (entre médicos, enfermeiros, psicólogos e voluntários) pelos eventos vividos no ambiente da UTI, constituindo da liberdade (autonomia, diferenciação) do paciente. 0 objetivo principal foi verificar o papel das percepções sociais construídas por pacientes para a avaliação do stress, levando em conta o papel dos personagens ligados à situação de saúde/doença considerados como responsáveis, ou não, pelo stress.

Os problemas de doença e morte sempre afetaram a humanidade, gerando comportamentos e pensamentos que fazem parte de tradições e costumes socioculturais, alguns prevalecendo até hoje (Herzlich \& Pierret, 1988; Parsons, 1951). Historicamente, houve uma grande mudança tanto da vida social quanto na prevenção e tratamento das doenças, a partir de uma crescente tomada de consciência do papel ativo dos indivíduos na manutenção de sua saúde (Flick, 1994). Mesmo assim, ainda existem em muitos ambientes sociais noções e práticas referentes à saúde e à doença que supõem o paciente como alguém passivo, a ser cuidado por profissionais, que têm nas mãos o seu destino e sorte, uma vez que a situação de atendimento hospitalar é fortemente marcada por normas específicas do ambiente que incluem hierarquias de poder informativo e de ação. Assim, o modo de lidar com a situação hospitalar adotado por pacientes é o misto de um repertório de experiências informativas e práticas anteriores, vivenciadas atualmente na situação da UTI (Cardoso \& Gomes, 2000; Gomes, Mendonça \& Pontes, 2002).

O stress é visto atualmente pela Organização Mundial de Saúde (Lipp, 2004) como um dos principais distúrbios contemporâneos, atingindo grande contingente de pessoas no globo terrestre, e está associado a uma gama enorme de doenças, absenteísmo e custos elevados na assistência médica.

Muito se tem discutido sobre o perfil dos pacientes das UTI, e percebe-se que muitos pacientes mantêmse lúcidos e acordados neste processo de internação, sofrendo males biológicos e também psicológicos/ psicossociais. Muitas destas vivências de stress, sensação de morte, sentimentos de culpa, ansiedade e depressão, entre outras, não são facilmente expressas e, conseqüentemente, não são detectadas nem pelos familiares, tampouco pela equipe, podendo o psicólogo funcionar como mediador neste processo de adoecimento, evitando-o e/ou minimizando-o.

O comportamento dos pacientes graves na UTI indica existirem conexões entre aspectos físicos, 
emocionais e sociais como variáveis intervenientes no processo de tratamento, recuperação e cura das enfermidades, assim como a vivência da situação de stress (Belkiss, 1999; Lusk \& Lash, 2005; Torres, 1998).

Dentro de uma vivência hospitalar, este estudo visou ampliar este olhar, buscando as percepções sociais tanto do ambiente físico quanto social. Para tanto, os profissionais que atuam na UTI foram vistos como personagens enquanto variáveis intervenientes (uma variável humana intermediária), podendo funcionar como indicador do tipo de interação hospitalar entre pacientes e familiares e os membros do staff (profissionais de saúde), dispondo e exercendo diferentes formas de poder social sobre os mesmos (French \& Raven, 1969).

A escolha de um personagem considerado mais ou menos responsável pelo stress vivido por parte de um paciente no ambiente da UTI pode indicar maior ou menor grau de autonomia individual, e ter conseqüências psicossociais sobre a experiência do mesmo. O interesse deste estudo recaiu sobre a comparação de avaliações das fontes de stress, supostamente originadas em personagens da equipe cuidadora. Existem as dimensões puramente físicas, mentais e comportamentais do indivíduo, assim como outras fontes de stress que são pertinentes às relações interindividuais e intergrupais, as quais dizem respeito a vivências representadas por pacientes, sendo afetadas pelo tipo de relação social. Contudo, todas as dimensões mencionadas tratam, de uma maneira ou outra, de negociação de conflitos entre o paciente e os diferentes personagens da equipe médica. Uma queixa de dor ou mal-estar vividos individualmente pode não ser atendida ou interpretada adequadamente por parte do staffe demais envolvidos, passando a ser objeto de um possível trabalho de convencimento, persuasão ou dissuasão por parte do paciente, cujo resultado afeta sua experiência de stress.

Apesar das particularidades da situação de UTI, pode-se supor que existem padrões construídos de interação entre paciente e profissional a serem mais conhecidos. Em uma primeira etapa, caberia saber o modo de inserção do paciente como alguém autônomo, diferenciado e com espaço privado demarcado no ambiente hospitalar em geral. Tal postura do paciente Ihe permitiria uma interação com os personagens da equipe cuidadora de modo mais direto, de igual para igual, apesar das dificuldades circunstanciais, tais como as dimensões físicas, mentais, informativas, entre outras.

Os profissionais poderiam servir como mediadores e, possivelmente, estimuladores para relações de igualdade, sem hierarquizações ou, contrariamente, como fortalecedores de desigualdade e dependência dentro destes ambientes. Em particular, foi problematizado o modo da percepção social da inserção do psicólogo no ambiente hospitalar, com base no pressuposto de que a menção espontânea dos profissionais da saúde e outros personagens, incluindo psicólogos, estando associados ou dissociados ao stress em diferentes contextos da vivência de internação hospitalar, permitiria fazer um mapeamento da situação e avaliar as possibilidades atuais de atuação profissional do psicólogo no ambiente hospitalar.

Sendo assim, na problemática da pesquisa realizada estão presentes dois conjuntos de fenômenos: a questão dos conhecimentos/avaliações dos pacientes, feitas a partir de percepções construídas sobre o ambiente físico e social do profissional no ambiente da UTI, e o stress

\section{Método}

A pergunta básica que norteou a pesquisa foi a respeito de possíveis papéis atribuídos por pacientes aos personagens da equipe, incluindo profissionais e não profissionais, na manutenção ou não do stress na UTI.

\section{Participantes}

Os pacientes foram de diferentes faixas etárias, todos maiores de idade, de ambos os sexos, de diferentes etnias, classes e grupos sociais. Os pacientes eram internos da UTI de um hospital privado no interior do Rio de Janeiro, apresentando uma saúde delicada, mas que não os impedia de responder ao inventário no local. A população investigada ( $n=85$ ) foi de $20 \%$ do total de pacientes internados em UTI, considerados segundo critérios específicos: pacientes lúcidos, com capacidade de verbalização e expressão inteligível, com no mínimo dois dias de internação em UTI (Soehren, 1995). A amostra compôs-se de homens $(n=50)$ e mulheres 
( $n=35)$, em uma faixa etária que variou de 14 a 87 anos, de diferentes estados civis, ocupações profissionais, naturalidades, patologias e escolaridades.

\section{Instrumento e procedimento}

O método utilizado neste projeto foi exploratório, com delineamento transversal, por se estender pelo período de 2002 a 2003, apresentando diferentes participantes ao longo do estudo.

Foi utilizado um inventário, a Escala de Stress em Unidade de Terapia Intensiva (ICUESS) (Soehren, 1995), para aferir os possíveis estressores destes pacientes por meio de suas vivências dentro da UTI. A tradução seguiu os padrões de validação interna na forma de Back Translation, com um profissional que traduziu para o Português e depois para o Inglês, avaliando a consistência da tradução. Após esta fase, o instrumento foi submetido a pesquisadores, conhecedores dos métodos psicométricos, e profissionais que atuam neste segmento hospitalar, que os avaliaram e propuseram algumas modificações frente aos inventários originais, sendo os mesmos re-avaliados e aprovados na forma como foram aplicados.

O referido inventário é composto por 40 afirmações, com uma escala do tipo Likert de graduação de intensidade que varia do 1 (=não estressante) ao 4 (=muitíssimo estressante), que também se baseou em um outro estudo (Soehren, 1995). A escala utilizada sofreu alterações e passou a apresentar 53 itens, adequandose melhor à realidade brasileira, com a inclusão do item 0 (=não ocorrido) (Anexo 1). Os participantes responderam segundo uma nova escala de graduação de intensidade do tipo Likert ( $0=$ não ocorrido, $1=$ não estressante, 2 = pouco estressante, $3=$ bastante estressante, 4= muitíssimo estressante), em relação aos estressores experimentados em determinadas situações dentro do ambiente da UTI. Este inventário não se propôs medir o stress vivenciado na unidade, pois seus itens constitutivos não são os melhores indicadores para avaliá-lo.

O questionário só foi aplicado após a informação dos objetivos do trabalho aos participantes (pacientes) e a obtenção da anuência dos mesmos. Foi solicitado que respondessem ao inventário sobre os estressores hospitalares por meio da escala de graduação de intensidade. Foi oferecido auxílio no preenchimento do inventário, ficando a cargo do participante respondê-lo segundo suas opiniões.

Ademais, o inventário ICUESS permitiu que os participantes identificassem, espontaneamente, os personagens da equipe considerados como responsáveis pelo stress vivido. Com estes dados, utilizou-se um método qualitativo de organização, a análise categorial temática, formando categorias de profissionais e demais personagens da equipe cuidadora, que foram tratadas estatisticamente. As categorias geradas foram: Médico, Enfermagem, Psicólogo, Equipe e Outros.

\section{Análise de dados}

Os resultados obtidos foram analisados pelo programa SPSS for Windows versão 10.0, dando ênfase à estatística descritiva de alguns dados sociográficos que compõem e delineiam a amostra (freqüências, porcentagens, médias, medianas, desvio-padrão, alfa de Crombach's, entre outras), além de testes de Correlação Linear de Pearson e Qui-quadrado.

Trabalhou-se, inicialmente, com os dados em função da mediana (menor e maior grau de stress). Em seguida, para aplicar os testes de correlação, criaram-se variáveis novas, segundo análise de conteúdo manifestado em cada afirmativa, como se segue: Individual, Interindivual e Intergrupal, que originaram os nomes das categorias utilizadas.

A categoria Individual reuniu afirmativas de cunho relativo ao paciente e sua vivência; a categoria Interindividual tratou de afirmativas entre o paciente e outros personagens não da equipe profissional; e a categoria Intergrupal referiu-se aos pacientes e profissionais e suas relações, como se segue: Individual, desmembrados em dois tipos de contexto: A) mental (perguntas 4, 13, 14, 16, 19, 20, 24, 36, 43, 47, 48) e B) física (perguntas 1, 2, 3, 6, 7, 8, 9, 10, 12, 17, 21, 22, 26, 28, 29, 32, 37, 41, 42, 45, 51, 53); Interindividual (interações entre pacientes e outros), subdividido em: A) encontros familiares - com parentes/íntimos e amigos (perguntas $5,27,44)$ e B) encontros não familiares - com outros pacientes e estranhos (perguntas 15, 46); Intergrupal (interações entre o paciente e alguém da equipe profissional), subdividido em: A) mental (perguntas 11, $18,23,25,30,31,34,35,39,40,49,50,52)$ e B) física (perguntas 33, 38). 
Por último, foram feitas correlações entre as categorias novas mencionadas em função de médias aritméticas, assim como testes de Qui-quadrado comparando grupos de profissionais e freqüências das categorias novas criadas.

\section{Resultados}

O inventário ICUESS apresentou um alfa de Cronbach $=0,8968$, demonstrando alta confiabilidade em seus itens como foram apresentados, com uma ocorrência de 85 respostas aos 53 itens pertencentes ao inventário. Ele apresentou média= 1,2295 e mediana $=1,1698$, com pequeno desvio padrão $(0,4234)$.

As situações geradoras de stress que foram encontradas neste estudo estavam relacionadas à ociosidade, perda da autonomia e sensação de abandono pela família. Em contrapartida, observou-se que as que menos incomodaram foram as de cunho relacional, nos cuidados assistenciais prestados, na atenção dispensada, entre outras.
Também para este inventário realizou-se uma reorganização das respostas espontâneas a respeito dos profissionais e demais personagens da equipe considerados relacionados ao stress, criando assim três novas categorias: individual (alfa $=0,8728)$, interindividual (alfa $=0,5281$ ) e intergrupal (alfa=0,5846); a primeira subdividiu-se em mental (alfa=0,7081) e físico (alfa=0,8068), a segunda em familiar ( $a l f a=0,5182$ ) e não familiar ( $a l f a=0,3848)$, e a terceira em mental (alfa=0,5753) e físi$\operatorname{co}(\mathrm{alfa}=0,1667)$.

Nas análises feitas em relação às categorias individual, interindividual e intergrupal, percentuais expressivos foram gerados no que tange às correlações positivas, embora a aplicação de testes de Qui-quadrado não tenha oferecido resultados significativos (Tabela 1). Na categoria individual, obteve-se de forma significativa a equipe (57\%) e médico (29\%) como personagens relacionados a um maior grau de stress (Tabela 2). Já quanto à categoria interindividual, observou-se a equipe (43\%) e a enfermagem (29\%) como principais personagens associados ao grau de stress (Tabela 3). A categoria intergrupal, finalmente, apresentou vários personagens,

Tabela 1. Descrição dos profissionais indicados na Correlação Linear de Pearson associados a um maior nível de stress. Universidade Federal do Rio de Janeiro, 2005.

\begin{tabular}{|c|c|c|c|c|}
\hline & Item Inventário & Personagem & Correlaçã & Linear \\
\hline \multirow[t]{2}{*}{ Individual mental } & Não saber quando os procedimentos vão ser realizados com você & Enfermagem & $\begin{array}{l}\text { Pearson } \\
\text { Sig. }\end{array}$ & $\begin{array}{l}0,229 \\
0,035\end{array}$ \\
\hline & & Equipe & $\begin{array}{l}\text { Pearson } \\
\text { Sig. }\end{array}$ & $\begin{array}{l}0,316 \\
0,003\end{array}$ \\
\hline \multirow[t]{8}{*}{ Intergrupal mental } & Ser acordado por membros da equipe & Equipe & $\begin{array}{l}\text { Pearson } \\
\text { Sig. }\end{array}$ & $\begin{array}{l}0,214 \\
0,049\end{array}$ \\
\hline & Excesso de pressa nos cuidados da equipe & Enfermagem & $\begin{array}{l}\text { Pearson } \\
\text { Sig. }\end{array}$ & $\begin{array}{l}0,243 \\
0,025\end{array}$ \\
\hline & Membros da equipe falando palavras que você não entende & Médico & $\begin{array}{l}\text { Pearson } \\
\text { Sig. }\end{array}$ & $\begin{array}{c}0,426 \\
0\end{array}$ \\
\hline & Não ter explicações sobre o tratamento & Equipe & $\begin{array}{l}\text { Pearson } \\
\text { Sig. }\end{array}$ & $\begin{array}{c}0,416 \\
0\end{array}$ \\
\hline & & Psicólogo & $\begin{array}{l}\text { Pearson } \\
\text { Sig. }\end{array}$ & $\begin{array}{l}0,214 \\
0,049\end{array}$ \\
\hline & Sentir que as pessoas estão mais atentas aos aparelhos do que a você & Enfermagem & $\begin{array}{l}\text { Pearson } \\
\text { Sig. }\end{array}$ & $\begin{array}{l}0,214 \\
0,049\end{array}$ \\
\hline & Membros da equipe não terem se apresentado a você & Enfermagem & $\begin{array}{l}\text { Pearson } \\
\text { Sig. }\end{array}$ & $\begin{array}{l}0,214 \\
0,049\end{array}$ \\
\hline & & Equipe & $\begin{array}{l}\text { Pearson } \\
\text { Sig. }\end{array}$ & $\begin{array}{l}0,346 \\
0,001\end{array}$ \\
\hline
\end{tabular}


Tabela 2. Freqüências significativas para a categoria Individual dos personagens da equipe considerados responsáveis pelo stress vivido. Universidade Federal do Rio de Janeiro, 2005

\begin{tabular}{lcc}
\hline Categorias & Freqüências & $\%$ \\
\hline Equipe & 4 & 57 \\
Médico & 2 & 29 \\
Enfermagem & 1 & 14 \\
\hline Total & 7 & 100 \\
\hline$\chi^{2}$ não significativo. & &
\end{tabular}

Tabela 3. Freqüências significativas para a categoria Interindividual dos personagens da equipe considerados responsáveis pelo stress vivido. Universidade Federal do Rio de Janeiro, 2005.

\begin{tabular}{lcc}
\hline Categorias & Freqüências & $\%$ \\
\hline Equipe & 3 & 43 \\
Médico & 1 & 14 \\
Enfermagem & 2 & 29 \\
Outros & 1 & 14 \\
\hline Total & 7 & 100 \\
\hline$\chi^{2}$ não significativo. & &
\end{tabular}

Tabela 4. Freqüências significativas para a categoria Intergrupal dos personagens da equipe considerados responsáveis pelo stress vivido. Universidade Federal do Rio de Janeiro, 2005.

\begin{tabular}{lcc}
\hline Categorias & Freqüências & $\%$ \\
\hline Equipe & 5 & 33 \\
Médico & 2 & 13 \\
Enfermagem & 6 & 40 \\
Outros & 1 & 7 \\
Psicólogo & 1 & 7 \\
\hline Total & 15 & 100 \\
\hline$\chi^{2}$ não significativo. & &
\end{tabular}

\begin{tabular}{|c|c|c|}
\hline $\begin{array}{l}\text { eq } \\
\text { no } \\
\text { Rio }\end{array}$ & $\begin{array}{l}\text { rados respons } \\
\text { spitalar (UTI). } \\
005 \text {. }\end{array}$ & stress vivido \\
\hline Categorias & Freqüências & $\%$ \\
\hline Equipe & 12 & 41 \\
\hline Médico & 5 & 17 \\
\hline Enfermagem & 9 & 31 \\
\hline Outros & 2 & 7 \\
\hline Psicólogo & 1 & 4 \\
\hline Total & 29 & 100 \\
\hline
\end{tabular}

338 $\chi^{2}=14,966 ; \quad g L=6 ; p<0,0048$. distribuídos como se segue: enfermagem (40\%), equipe (33\%), psicólogo (7\%) e médico (13\%), como principais estressores (Tabela 4). Quando as freqüências dos personagens foram agrupadas, estas apresentaram índices altos; a equipe e a enfermagem tiveram um papel significativo, apresentando-se como estressores, neste momento, fato este ratificado pelo teste qui quadrado, que demonstrou uma distribuição de freqüências dos personagens mencionados estatisticamente significativa, segundo os conteúdos analisados (Tabela 5).

\section{Discussão}

Retomando a problemática central desta investigação, partiu-se da suposição de que parte dos fenômenos de vivência psicológica de stress é modificada por percepções sociais. Ou seja, tendo em vista a epistemologia do senso comum de que os fenômenos de saúde e doença dizem respeito ao orgânico (Herzlich, 1970), ainda não se dá importância ao papel da dimensão psicológica/psicossocial para a intensificação e/ou atenuação da vivência de stress, tanto ao nível dos aspectos físicos, quanto dos que envolvem relações sociais.

A díade saúde-doença é um processo dinâmico, complexo, com etiologia variável e com um indivíduo em processo de mudança, como bem salientou esta mesma autora (Herzlich, 1991), atentando para um reducionismo criticável. Os aspectos fisiológicos, psicológicos e sociais têm grande importância no processo de recuperação destes indivíduos. Tais dados se refletem no estudo aqui descrito, quando, nas correlações na categoria individual do inventário, ocorreu uma tendência a agrupar os personagens de forma generalista (equipe), sem atribuir responsabilidade a um personagem específico no momento de stress, tendência esta que pode estar ligada às relações de poder e influência exercida pela equipe de profissionais em um momento tão delicado. Existe uma hierarquização do poder, com diferentes saberes profissionais exercidos por autoridades diferentes nas relações intrahospitalares, que faz com que formas de submissão e dominação sejam instauradas. Assim, apontar a"equipe" como estressora seria uma maneira de evitar um confronto do paciente com personagens específicos 
considerados poderosos. Na relação equipe-paciente, repete-se uma forma de poder baseada na informação da equipe médica sobre quais sejam as necessidades do indivíduo, desprovido e fragilizado na situação de UTI (French \& Raven, 1969).

Nesse sentido, alguns estudos realizados a partir de referenciais teóricos da psicologia social cognitiva trataram deste tema (Aronson et al., 2002). Eles empregaram o conceito de controle percebido, que seria aqui definido como a crença de ser possível influenciar o ambiente de maneira a determinar se ocorrerão resultados positivos ou negativos. Ou seja, a interpretação pessoal atribuída à situação percebida e o controle exercido sobre ela funcionariam como fator minimizador de efeitos estressores. Em um destes estudos (Aronson et al. 2002), descobriu-se que mesmo entre os participantes que tinham grande controle da situação, e que suportavam bem as situações percebidas como estressoras, instaurava-se outro fenômeno, chamado desamparo aprendido. Dentro da realidade da UTI e dos achados do estudo aqui tratado, pode-se inferir que a sensação de abandono e as perdas recorrentes nas fases agudas da doença podem contribuir para a formação destefenômeno.

O psicólogo não foi apresentado como um personagem ligado ao stress em relação ao todo, mas apareceu como estressor ao nível intergrupal, ainda que em menor proporção que a enfermagem, equipe e médico. Ou seja, o psicólogo, por ter sido menos associado ao stress, poderia tornar-se um facilitador no manejo do mesmo. Para outros conjuntos de experiências hospitalares de ordem individual e interindividual, como já mencionado anteriormente, a equipe apareceu como grande personagem estressor, enquanto o médico figurou mais relacionado a aspectos individuais, e a categoria enfermagem, a aspectos interindividuais. Ou seja, pode-se inferir que o papel do psicólogo como possível atenuador de stress aos níveis individuais/ interindividuais ficou reduzido, uma vez que as percepções sociais, construídas e adotadas na experiência hospitalar, giraram em torno do cuidado corporal exercido diretamente pelos demais profissionais fora da psicologia, cabendo um trabalho do psicólogo para modificar tal situação, sob pena de diminuir os efeitos do seu trabalho. É preciso compreender estes resultados como produto de interações psicossociais entre mem- bros de diferentes grupos. Parece existir uma expectativa social de submissão de pacientes e familiares em relação aos profissionais de saúde (médicos e enfermeiros).

Outro aspecto salientado no estudo refere-se ao controle eficaz da dor e a importância de informar os pacientes sobre seu tratamento. Variáveis como comunicação eficaz, música para promover relaxamento, diminuição da sensação de isolamento (item este que foi indicado no estudo como estressor, causador de uma sensação de abandono familiar) e fotografias de parentes seriam contribuições possíveis para minimizar os impactos sofridos dentro de uma UTI. Esses últimos achados estão intimamente ligados às proposições apregoadas pelo processo de humanização destas unidades e pela própria bioética, visando ao afastamento do desamparo aprendido e de atribuições que levem a mais insegurança e despersonalização. No âmbito da sociedade, a divulgação de direitos do paciente deve fazer parte do exercício e fortalecimento da cidadania em geral.

É importante a realização de pesquisas futuras com pacientes e familiares sobre representações sociais de profissionais de saúde que atuam em hospitais, em seus diferentes setores. Tal trabalho poderia indicar, por exemplo, os critérios psicossociais usados por pacientes e familiares nas suas relações humanas no ambiente hospitalar, de modo mais extenso e rico em informações, o que permitiria preencher lacunas e diminuir limitações empíricas encontradas no estudo aqui relatado.

\section{Conclusão}

Apesar das limitações amostrais, esta investigação permitiu avançar no estudo do stress em ambiente de UTI. À guisa de conclusão, pode-se afirmar que os personagens da equipe foram percebidos como estressores. Entretanto, ocorreu uma tendência a diluir a responsabilidade dos profissionais e demais personagens específicos, principalmente quando associados a muito stress, transformando-se em um grupo único, possivelmente para evitar um confronto com os mesmos em situação de desequilíbrio frente ao poder de informação. Nesse sentido, as percepções sociais estudadas confirmaram a importância do psicólogo como mediador/facilitador das relações sociais no ambiente hospitalar, cabendo mais investigações. 
Enfim, sugere-se que a psicologia hospitalar tende a crescer cada vez mais na sociedade brasileira, em especial o trabalho em UTI, que deverá se tornar uma área de atuação importante entre os psicólogos. Nesse quadro, o paciente e seu processo de saúde e doença passam a ser vistos como um conglomerado de fatores que interferem em seu tratamento e recuperação.

\section{Referências}

Aronson, E., Akert, R.M., \& Wilson, T.D. (2002). Psicologia Social. Rio de Janeiro: LTC.

Belkiss, W. R. (1999). Princípios para prática da psicologia clínica em hospitais. São Paulo: Casa do Psicólogo.

Biaggi, T. M. (2002). A atuação do psicólogo hospitalar em unidade de terapia intensiva: adultos. São Paulo: Nêmeton - Centro de Estudos e Pesquisas em Psicologia e Saúde.

Cardoso, M. H. C. A., \& Gomes, R. (2000). Representações sociais e história: referenciais teórico-metodológicos para o campo da saúde coletiva. Cadernos Saúde Pública, 16 (2), 499-506.

Donahoe, M., \& Pinsky, M. (2002). Patients' recollections of stressful experiences while receiving prolonged mechanical ventilation in an intensive care unit. Critical Care Medicine, 30 (4), 746-752.

Flick, U. (1993). La perception de la santéet la maladie: théories subjectives et représentations sociale. Paris: L'Harmattan.

Flick, U. (1994). Social representations and the social construction of everyday knowledge: theoretical and methodological queries. Social Science Information, 33 (2), 179-197.

French Jr., J. R. P., \& Raven, B. (1969). As bases do poder social. In D. Cartwright \& A. Zander (Orgs.), Dinâmica de grupo:pesquisa e teoria, 2 (pp.758-779). São Paulo: Herder.
Gomes, R., Mendonça, E. A., \& Pontes, M. L. (2002). As representações sociais e a experiência da doença. Cadernos Saúde Pública, 18 (5), 1207-1214.

Herzlich, C. (1970). Médecine, Maladie et Société. Paris: Mouton.

Herzlich, C. (1973). Health and illness: a social psychological analysis. London: Academic Press.

Herzlich, C. (1991). A Problemática da Representação Social e sua Utilidade no Campo da Doença. Rio de Janeiro: Physis - Revista de Saúde Coletiva. 1 (2), 23-34.

Herzlich, C., \& Pierret, J. (1988). De ayer a hoy: construcción social del enfermo. Cuadernos Médico Sociales, 43, 21-30.

Lipp, M. E. N. (Org.). (2004). O stress no Brasil: pesquisas avançadas. Campinas: Papirus.

Lusk, B., \& Lash, A. A. (2005). The stress response, psychoneuroimmunology, and stress among ICU patients. Dimensions of Critical Care Nursing, 24 (1), 25-31.

Parsons, T. (1951). Social structure and dynamic process: the case of modern medical practice. In T. Parsons. The social system (pp.428-480). Glencoe, Illinois: The Free Press.

Rangé, B. (2001). O stress emocional e seu tratamento. Psicoterapias cognitivas: um diálogo com a psiquiatria (pp.473-490). Porto Alegre: Artmed.

Soehren, P. (1995). Stressors perceived by cardiac surgical patients in the intensive care unit. American Journal of Critical Care, 4 (1), 71-76.

Torres, W. C. (1998). A morte, o morrer e a ética. Rio de Janeiro: Científica Nacional.

Wagner, W., Duveen, G., Themel, M., \& Verma, J. (2000). The modernization of tradition: thinking about madness in Patna, India. Culture \& Psychology, 5 (4), 413-445.

Recebido em: 30/10/2007

Versão final reapresentada em: 26/11/2007

Aprovado em: 13/12/2007 
ANEXO

INVENTÁRIO DE ESTRESSORES EM UNIDADE DE TERAPIA INTENSIVA (ICUESS)

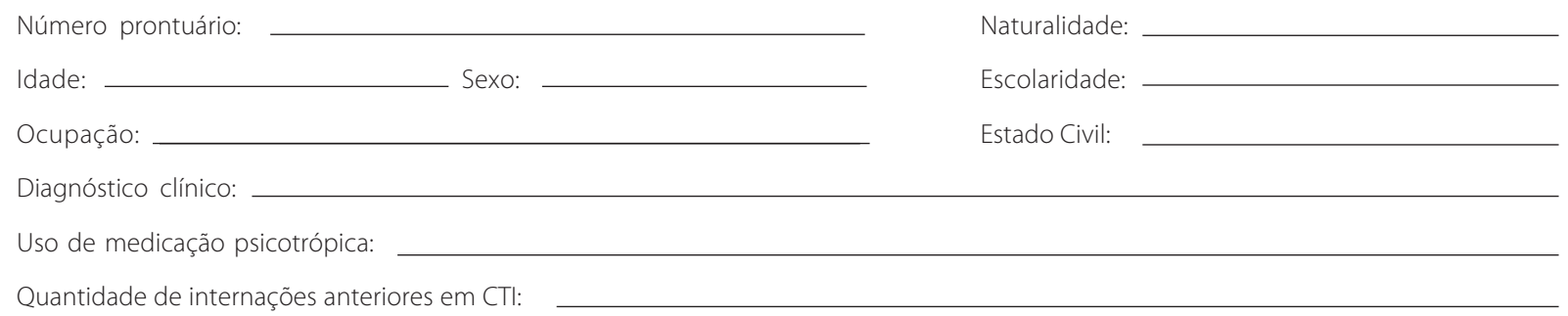

Solicitamos que você responda à seguinte lista de afirmativas segundo a escala que segue abaixo. Não há respostas certas ou erradas; desejamos saber, neste momento, o que Ihe causa incômodo. Em algumas afirmativas temos linhas nas quais deverá ser indicado o(s) membro(s) da equipe responsável pelo fato.

\section{$\begin{array}{lllll}\text { (0) Não ocorrido } & \text { (1) Não estressante } & \text { (2) Pouco estressante } & \text { (3) Bastante estressante } & \text { (4) Muitíssimo estressante }\end{array}$}

1. ( ) Ter tubos na boca e no nariz

2. ( ) Sentir-se preso pelos drenos e tubos

3. ( ) Sentir dor

4. ( ) Não ter controle sobre si mesmo

5. ( ) Sentir falta de seu marido ou esposa

6. ( ) Não dormir

7. ( ) Ter sede

8. ( ) Ser furado por agulhas

9. ( ) Não conseguir mover suas mãos e braços devido à linha venosa

10. ( ) Sensação de muito calor ou muito frio

11. ( ) Ser acordado por membros da equipe. Quem?

12. ( ) Existência de luzes acesas constantemente

13. ( ) Sentir solidão e tristeza

14.( ) Sentir-se chateado ou aborrecido

15. ( ) Ouvir o gemido de outros pacientes

16. ( ) Não saber quando os procedimentos vão ser realizados com você. Quem?

17. ( ) Cama/travesseiros não confortáveis

18. ( ) Excesso de pressa nos cuidados da equipe. Quem?

19. ( ) Não saber que dia é hoje

20. ( ) Não ter nenhuma privacidade

21. ( ) Não saber onde está agora

22. ( ) Ter que usar oxigênio

23. ( ) Membros da equipe falando palavras que você não entende. Quem?

24. ( ) Não saber que horas são

25. ( ) Não ter explicações sobre o tratamento. Quem?

26. ( ) Ter máquinas e aparelhos estranhos ao seu redor

27. ( ) Ver familiares e amigos durante poucos minutos por dia

28. ( ) Ouvir alarmes e buzinas dos aparelhos

29. ( ) Ouvir o telefone tocar

30. ( ) Ser examinado freqüentemente por membros da equipe. Quem?

31. ( ) Olhar bolsas e sacos pendurados sob a cabeça

32. ( ) Sentir cheiros desagradáveis ao seu redor

33. ( ) Ter membros da equipe constantemente fazendo procedimentos ao redor da sua cama. Quem?

34. ( ) Membros da equipe falando muito alto. Quem?

35. ( ) Ser examinado cada dia por um médico diferente 
ANEXO

INVENTÁRIO DE ESTRESSORES EM UNIDADE DE TERAPIA INTENSIVA (ICUESS)

36. ( ) Barulhos não familiares e estranhos

37. ( ) Ter que olhar constantemente para o teto

38. ( ) Ter sua pressão arterial verificada várias vezes

39. ( ) Sentir que as pessoas estão mais atentas aos aparelhos do que a você. Quem?

40. ( ) Membros da equipe não terem se apresentado a você. Quem?

41. ( ) Não se alimentar normalmente

42. ( ) Estar sem a prótese dentária

43. ( ) Estar sem suas roupas e objetos pessoais

44. ( ) Não ter o acompanhamento da família por mais tempo

45. ( ) Estar amarrado no leito. Quem?

46. ( ) Não ter como se comunicar

47. ( ) Não saber o que aconteceu com você. Quem?

48. ( ) Não ter o que fazer para ocupar seu tempo livre

49. ( ) Ser tratado como criança. Quem?

50. ( ) O uso de palavras tipo: "vozinho", "queridinho" para conversar com você. Quem?

51. ( ) Ter que fazer suas necessidades fisiológicas no leito

52. ( ) Ser visitado pelo seu médico assistente

53. ( ) Não poder ficar sentado no leito

(Traduzido e Adaptado de Cochran and Ganong por Leonardo Mello e colaboradores).

342 